University of Wollongong

Research Online

Faculty of Engineering and Information

Faculty of Engineering and Information

Sciences - Papers: Part A

Sciences

$1-1-2007$

\title{
The OFDM-IDMA approach to wireless communication systems
}

Li Ping

City University of Hong Kong

Qinghua Guo

City University of Hong Kong, qguo@uow.edu.au

Jun Tong

City University of Hong Kong

Follow this and additional works at: https://ro.uow.edu.au/eispapers

Part of the Engineering Commons, and the Science and Technology Studies Commons

Research Online is the open access institutional repository for the University of Wollongong. For further information contact the UOW Library: research-pubs@uow.edu.au 


\title{
The OFDM-IDMA approach to wireless communication systems
}

\author{
Abstract \\ This article outlines the basic principles of OFDM-IDMA. Comparisons with other alternative technologies \\ such as OFDM-CDMA and OFDMA are provided. Some attractive features of OFMD-IDMA are explained, \\ including low-cost iterative multi-user detection, flexible rate adaptation, frequency diversity, and \\ significant advantages regarding spectral and power efficiency. @ 2007 IEEE. \\ Keywords \\ communication, wireless, approach, systems, idma, ofdm \\ Disciplines \\ Engineering | Science and Technology Studies \\ Publication Details \\ Ping, L., Guo, Q. \& Tong, J. (2007). The OFDM-IDMA approach to wireless communication systems. IEEE \\ Wireless Communications, 14 (3), 18-24.
}




\title{
Next-Generation CDMA vs. OFDMA For \\ $4 G$ WIRELESS APPLICATIONS
}

\section{THE OFDM-IDMA APPROACH TO WIRELESS COMMUNICATION SYSTEMS}

\author{
LI PING, QINGHUA GUO AND JUN TONG \\ City UNIVERSITY OF HONg KONG, HONg KONg SAR
}

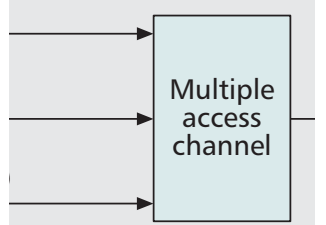

(a) IDMA

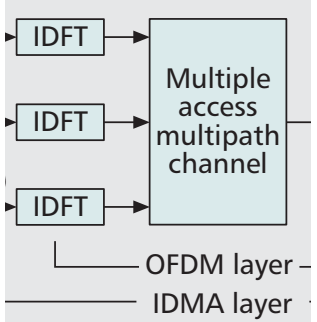

(b) OFDM-IDMA

The authors outline the basic principles of OFDM-IDMA.

They provide comparisons with other alternative technologies such as OFDM-CDMA and OFDMA, and they explain some of the attractive features of OFMD-IDMA.

\section{ABSTRACT}

This article outlines the basic principles of OFDM-IDMA. Comparisons with other alternative technologies such as OFDM-CDMA and OFDMA are provided. Some attractive features of OFMD-IDMA are explained, including lowcost iterative multi-user detection, flexible rate adaptation, frequency diversity, and significant advantages regarding spectral and power efficiency.

\section{INTRODUCTION}

Frequency division multiple access (FDMA), time division multiple access (TDMA), and code division multiple access (CDMA) are the main technologies used in first- to third-generation mobile cellular networks. Much recent research has been focused on the evolution paths for beyond third-generation (B3G) and fourth-generation $(4 \mathrm{G})$ systems. Orthogonal frequency division multiplexing (OFDM) and the related orthogonal frequency division multiple access (OFDMA) have been widely studied for this purpose. OFDMA offers a number of advantages. Some of these are listed below. (Here the focus is on comparison between OFDMA and CDMA, since these are the two main options being considered for $\mathrm{B} 3 \mathrm{G}$ and $4 \mathrm{G}$.)

- Based on a cyclic prefix technique, OFDMA can achieve inter-symbol interference (ISI) free transmission in multi-path channels. The related receiver cost is independent of the number of resolvable paths. However, CDMA employs a rake receiver technique to suppress multi-path effects and the related cost increases at least linearly with the number of paths and so can be very high for channels with a large number of paths.

- OFDMA allows each user to select subcarriers with high channel gains. This provides a means to realize the water-filling principle - a wellknown strategy in information theory for approaching the ISI channel capacity. However, it is not convenient to realize the water-filling principle by CDMA.
- OFDMA is in principle an orthogonal technique. Multiple access interference (MAI) free transmission can be achieved by allocating different subcarriers to different users. In contrast, MAI among same-cell users is a serious issue in CDMA.

- Future systems may need to support both packet data and speech services. Packet mode service potentially involves high-rate transmission with all of the bandwidth resource allocated to a single user (or a small number of users), which is relatively easy for OFDMA but not for CDMA. Due to its spread spectrum nature, CDMA is not convenient for high-rate transmission by a single user (or a small number of users). ${ }^{1}$

Plain OFDMA is prone to fading in subcarriers. One solution to this problem is the use of forward error control (FEC) coding across subcarriers. Another solution is OFDM-CDMA, in which each information bit is spread and transmitted over a number of subcarriers. Since spreading results in rate loss, OFDM-CDMA allows multiple users to share common subcarriers. This avoids rate loss but at the cost of reintroducing MAI.

Multi-user detection (MUD) is a potential solution to the MAI problem. In the past, MUD was viewed as a costly option, but the situation has changed recently with progress in iterative processing techniques [1]. In particular, the interleave division multiple access (IDMA) scheme [2-4] allows a very low-cost chip-by-chip (CBC) MUD algorithm to be used. The related complexity (after being normalized to each user) is independent of the number of users, indicating its potential for practical use. A disadvantage

${ }^{1}$ Note that we should distinguish single-user rate and cellular spectral efficiency. The latter is measured by the aggregate rate of all the users. When a large number of users are present, the CDMA cellular spectral efficiency can be very competitive compared with other alternatives such as TDMA or FDMA. However, since CDMA involves spreading, its achievable maximum single-user rate is relatively low. 
of IDMA is that its receiver complexity still increases linearly with the number of paths, which can be a concern for very wideband systems.

The OFDM-IDMA scheme presented in [5, 6] combines most of the advantages of the multiple access schemes mentioned above (such as OFDMA, CDMA, OFDM-CDMA, and IDMA) and avoids their individual disadvantages. With OFDM-IDMA, ISI is resolved by an OFDM layer and MAI is suppressed by an IDMA layer, both at low cost. With these two main obstacles removed, OFDM-IDMA offers many attractive features including:

- The achievable throughput of OFDM-IDMA is considerably higher than that reported for CDMA and OFDM-CDMA in the literature.

- If the entire bandwidth resource in a cell can be allocated to a single user, OFDM-IDMA can achieve a very high single-user throughput using a superposition coding technique [8]. This property is crucial for packet mode transmission. It is difficult to achieve very high single-user throughput with the existing CDMA or OFDM-CDMA techniques.

- The power efficiency of OFDM-IDMA can be greatly enhanced by use of an unequal power control strategy inherited from IDMA [2].

- In fading environments, OFDM-IDMA can offer the so-called multi-user gain [7], which is an interesting concept from information theory. The power efficiency of OFDM-IDMA is potentially much higher than that of orthogonal schemes such as FDMA, TDMA, or OFDMA. The difference can be very significant at high rates (Fig. 5).

In what follows, we outline the basic principles of OFDM-IDMA. Comparisons are made between OFDM-IDMA and other alternatives. We demonstrate the advantages of OFDMIDMA using numerical examples. We also discuss existing issues and future research topics.

\section{OFDMA AND OFDM-CDMA PRINCIPLES}

OFDMA is an orthogonal multiple access scheme directly based on OFDM techniques, which is formed by dividing the available subcarriers in OFDM into non-overlapping subsets and assigning each user a unique subset. Some properties of OFDMA are listed below

- Each subcarrier is occupied by at most one user.

- Orthogonality among subcarriers can be maintained in multi-path channels provided that the cyclic prefix length is longer than the channel length. Clearly, this also guarantees the orthogonality among users.

- In practice, DFT and IDFT can be implemented using the fast Fourier transform (FFT) and its inverse IFFT with greatly reduced cost.

- Each coded bit is modulated onto a subcarrier by IFFT. All of the modulated sub-carries are transmitted in parallel.

If channel information is known at the transmitter side, a proper strategy is to assign each user subcarrier with relatively high gains. (Note that channel gains seen by different users are different.) This agrees with the well known water-filling principle for achieving the capaci- ty of a frequency selective channel. However, when no channel information is available at the transmitter side, the performance of plain OFDMA may be affected by fluctuation (i.e., fading) among the gains of subcarriers. This problem motivates the work on OFDMCDMA.

Similar to OFDMA, the information in OFDM-CDMA [9-12] is also modulated onto the subcarriers by using IDFT. We highlight the main characteristics of OFDM-CDMA below.

- For OFDM-CDMA, each coded bit is spread into several chips before being modulated onto different subcarriers. This results in frequency diversity that averages out the fading effect.

- However, the spreading operation results in redundancy. To avoid rate loss, OFDMCDMA allows several users to share a common subcarrier. More specifically, the restriction in OFDMA that each subcarrier is occupied by at most one user is removed in OFDM-CDMA, which, unfortunately, reintroduces the MAI problem. ${ }^{2}$

- The use of the OFDM in OFDM-CDMA resolves the ISI problem. When the channel length is very large (such as for a very wideband system), OFDM-CDMA can be more efficient than CDMA with regard to receiver complexity, since the complexity of an OFDMCDMA receiver is independent of the channel length while that of a CDMA rake receiver increases at least linearly with the channel length.

MAI imposes the main limitation on OFDMCDMA performance. Single-user detection results in poor performance. MUD can be used to alleviate this problem, but the complexity of the conventional MUD techniques constitutes an obstacle in their practical use. For example, the well known linear minimum mean square error (MMSE) MUD technique [13] has complexity $O\left(K^{2}\right)$ per user, which causes concern when the number of users $K$ is large. Other options based on interference cancellation techniques have been studied [10-12] for different performancecomplexity trade-offs.

\section{IDMA PRINCIPLES}

In IDMA [2-4] each user is assigned a unique interleaver, which is used to distinguish it from other users. IDMA can be regarded as a special form of CDMA. An attractive feature of IDMA is that it allows the use of a low-complexity iterative MUD technique that is applicable to systems with a large number of users.

The basic principles of IDMA are illustrated in Fig. 1a. At the transmitter side, the information data from user $k$ is first encoded by an FEC encoder labeled by ENC. The resultant signal is then interleaved by its unique interleaver $\pi_{k}$ before transmission. Let us consider a flat fading channel (i.e., single-path channel). The received signal can be written as

$$
\begin{aligned}
r(n) & =\sum_{k=1}^{K} h_{k}(n) x_{k}(n)+z(n) \\
& =h_{k}(n) x_{k}(n)+\xi_{k}(n),
\end{aligned}
$$

MAl imposes the

main limitation on OFDM-CDMA performance. Single-user detection results in poor performance. MUD

can be used to alleviate this problem, but the complexity of the conventional MUD techniques constitutes an obstacle in their practical use.

\footnotetext{
2 When the number of users is less than the spreading length, orthogonal spreading sequences can be used, but frequency selective fading may destroy the orthogonality.
} 
With IDMA, chip-level interleaving ensures that the transmitted sequences from different users are almost un-correlated. Consequently, matrix operations are not necessary and the simple CBC detection is sufficient to provide near optimal performance.

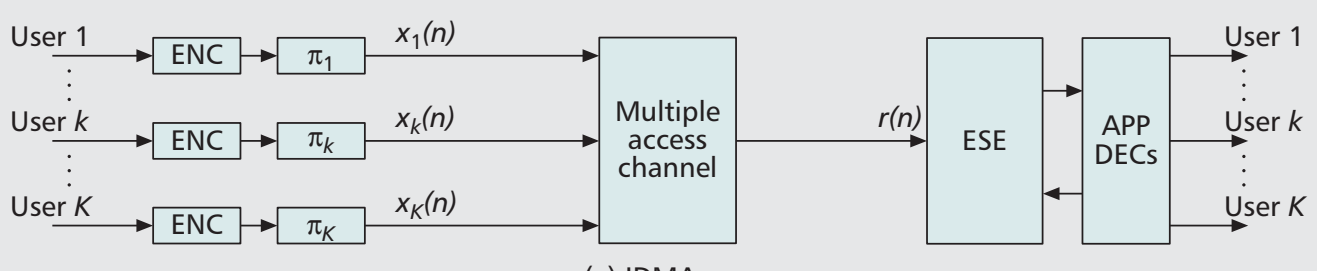

(a) IDMA

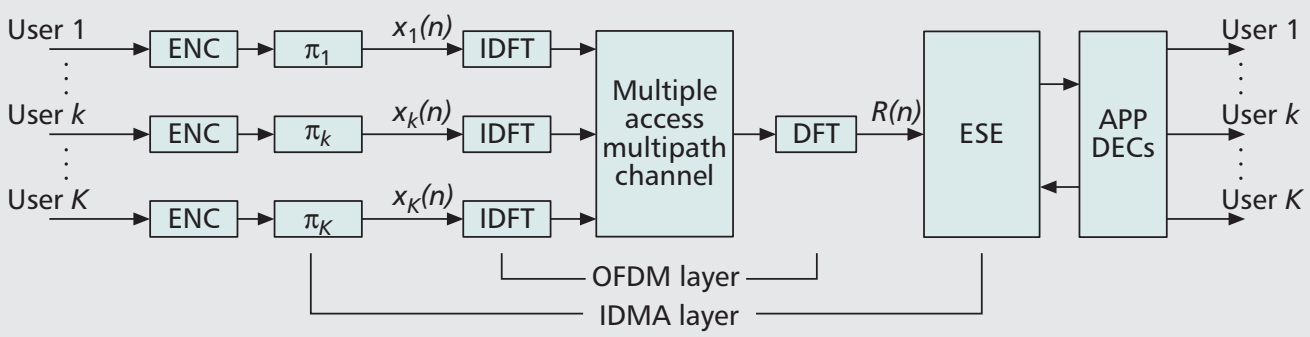

(b) OFDM-IDMA

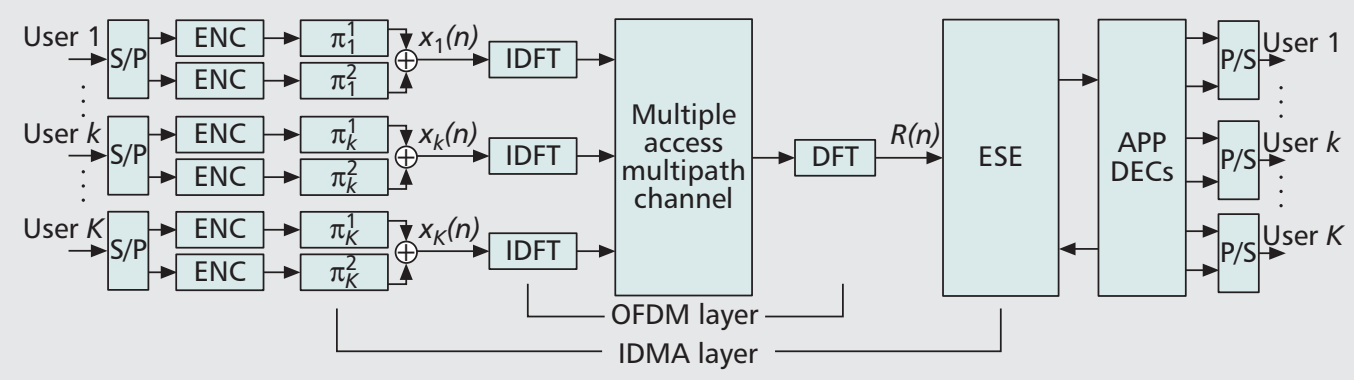

(c) Multi-stream OFDM-IDMA

Figure 1. Illustration of various multiple access schemes: a) IDMA; b) OFDM-IDMA; and c) multistream OFDM-IDMA. Cyclic prefix insertion and removal are not explicitly shown in $(b)$ and $(c)$. $S / P$ and $P / S$ stand for serial-to-parallel and parallel-to-serial converters respectively.

where $h_{k}(n)$ is the fading coefficient seen by user $k$ at time $n,\{z(n)\}$ are samples of additive white Gaussian noise (AWGN), and $\xi_{k}(n)=\Sigma_{m \neq k}$ $h_{m}(n) x_{m}(n)+z(n)$ represents the interference plus noise component in $r(n)$ with respect to user $k$ at time $n$. From the central limit theorem, $\xi_{k}(n)$ can be approximated by a Gaussian random variable. The core of the IDMA receiver in Fig. 1a consists of the iteration of the following two operations.

- Estimate $x_{k}(n)$ based on Eq. 1b. This is a standard signal detection problem since Eq. $1 \mathrm{~b}$ is in a signal plus noise form. It is carried out by the elementary signal estimator (ESE) shown in Fig. 1a. Under the Gaussian approximation, $\xi_{k}(n)$ is completely characterized by its mean $E\left(\xi_{k}(n)\right)$ and variance $\operatorname{Var}\left(\xi_{k}(n)\right)$. A fast technique is described in [2] to compute $E\left(\xi_{k}(n)\right)$ and $\operatorname{Var}\left(\xi_{k}(n)\right)$.

- Process the outputs of the above operation using the a posteriori probability decoders (APP DECs). The results are used to refine the estimates of the mean and variance of $\xi_{k}(n)$ to be used in the next iteration.

In MUD for conventional CDMA systems, matrix operations are required to handle the correlation among the spreading sequences of different users [13]. With IDMA (and OFDM-IDMA discussed in the next sub-section), chip-level interleaving ensures that the transmitted sequences from different users are almost un-correlated. Consequently, matrix operations are not necessary and the simple CBC detection outlined above (with cost independent of $K$ ) is sufficient to provide near optimal performance. This removes the main obstacle to MUD in applications.

\section{OFDM-IDMA PRINCIPLES}

The IDMA receiver complexity over multi-path channels is related to the channel length. Recently, OFDM-IDMA was proposed [5, 6] as an alternative to plain IDMA over multi-path channels. OFDM-IDMA inherits most of the merits of OFDM and IDMA. The key advantage of OFDM-IDMA is that MUD can be realized efficiently with complexity per user independent of the channel length and the number of users, which is significantly lower than that of other alternatives.

Figure $1 \mathrm{~b}$ shows the transmitter/receiver structure of an OFDM-IDMA system with $K$ users. The coded signals are first interleaved by user-specific interleavers $\left\{\pi_{k}\right\}$. Then the resultant signals, again denoted by $\left\{x_{k}(n)\right\}$, are modulated onto subcarriers by using IDFT. Each subcarrier can be occupied by several users, so users are solely distinguished by their interleavers. The received signal after DFT can be represented by 


$$
\begin{aligned}
R(n) & =\sum_{k=1}^{K} H_{k}(n) x_{k}(n)+Z(n) \\
& =H_{k}(n) x_{k}(n)+\Xi_{k}(n),
\end{aligned}
$$

where $H_{k}(n)$ is referred to as the channel gain of the $n$th subcarrier for user $k, Z(n)$ denotes AWGN at subcarrier $n$, and $\Xi_{k}(n)=\Sigma_{m \neq k}$ $H_{m}(n) x_{m}(n)+Z(n)$ represents the interference plus noise component in $R(n)$ with respect to user $k$ at subcarrier $n$. From the central limit theorem, $\Xi_{k}(n)$ can again be approximated by a Gaussian random variable. The similarity between Eqs. 1 and 2 indicates that the receiver principles outlined for IDMA earlier can be directly applied to OFDM-IDMA.

Suppose that the aggregate rate $R$ in Fig. $1 \mathrm{~b}$ is fixed and each user has a single-user rate $R / K$. When $K$ is large, the single-user rate $R / K$ will be low. A simple and convenient way to realize a low-rate code is concatenating a common FEC code (such as a rate-1/2 convolutional or turbo or LDPC code) with a repetition code. In this case, the repetition coding acts similarly as the spreading operation for CDMA. The outputs of a repetition encoder are dispersed over different subcarriers after IDFT. At the receiver, the inputs to a repetition decoder are collected from different subcarriers and combined. As a result, the frequency selective part of fading (i.e., the difference among the gains of different subcarriers) is averaged out when the rate of repetition coding is sufficiently low (e.g., $\leq 1 / 8$ ), and the average channel gains of subcarriers become the dominating factor, hence achieving frequency diversity.

The above also implies a fast technique to predict the performance of OFDM-IDMA based on knowledge of the average channel gains. Figure 2 shows the simulated and predicted performance of OFDM-IDMA using a rate- $1 / 2$ convolutional code (with generator $\left.(23,35)_{8}\right)$ followed by a repetition code with different length $S$. QPSK modulation is used. The average channel gains are used for prediction. ${ }^{3}$ The number of users $K=2 \mathrm{~S}$, so the system throughput is kept constant $(R=2)$. We can see from Fig. 2 that the predicted and simulated performance curves are quite close when $S \geq 8$.

The availability of a fast performance prediction technique is crucial for search-based system optimization. In particular, it has been shown in [2] that the spectral and power efficiency of IDMA can be greatly enhanced by using an unequal power allocation strategy. The same principle also applies to OFDM-IDMA and the optimization techniques developed in [2] can be used. The optimized OFDM-IDMA scheme possesses several attractive properties, including

- Very high spectral efficiency

- Flexibility in multi-user as well as single-user mode transmission,

- Multi-user gain in fading channels

${ }^{3}$ Specifically, for a channel defined in Eq. 2, denote by $\eta_{\mathrm{k}}$ the average of $\left\{\left|\mathrm{H}_{\mathrm{k}}(\mathrm{n})^{2}\right|, \forall \mathrm{n}\right\}$ for user $\mathrm{k}$. We construct a companion system in which user $\mathrm{k}$ sees a quasi-static flat fading channel with channel (power) gain $\eta_{\mathrm{k}}$. The prediction is done by applying an SNR evolution technique (following the discussions in [2] and considering the impact of fast fading on DECs) to this companion system.

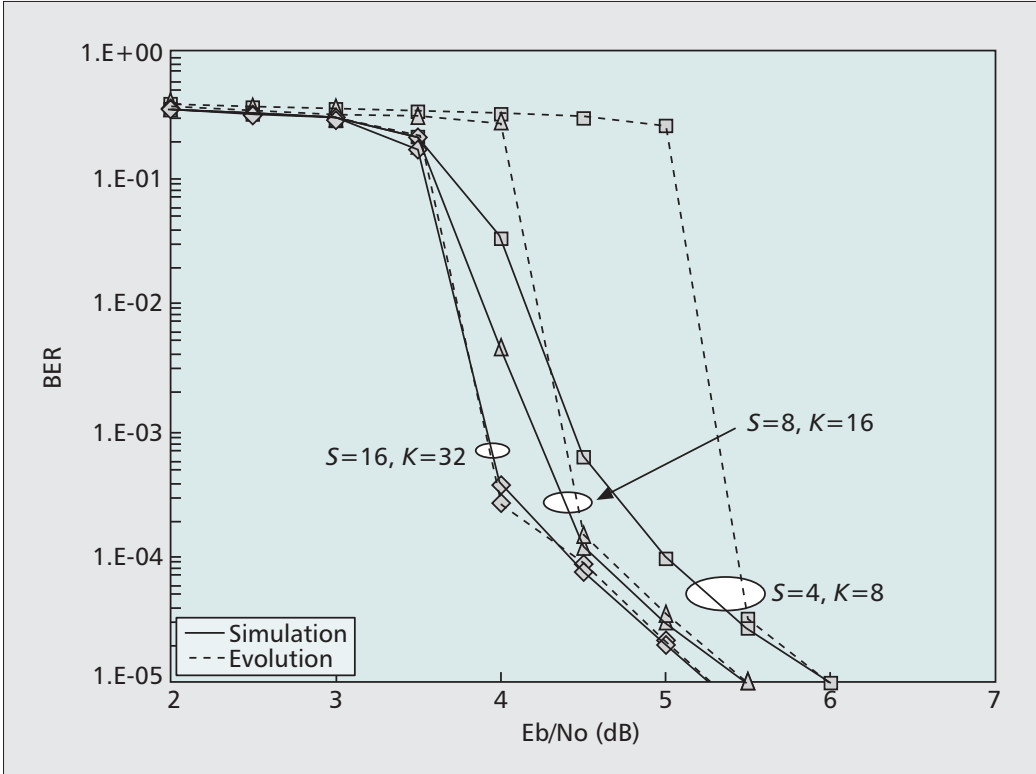

Figure 2. The predicted and simulated performance curves for OFDMIDMA. In this example, we assume that $\left\{\mathrm{H}_{\mathrm{k}}(\mathrm{n}), \forall \mathrm{k}, \forall \mathrm{n}\right\}$ are uncorrelated and identically Gaussian distributed [10], and each user has unit average channel (power) gain. Information bit length $=512$, and iteration number $=$ 15 .

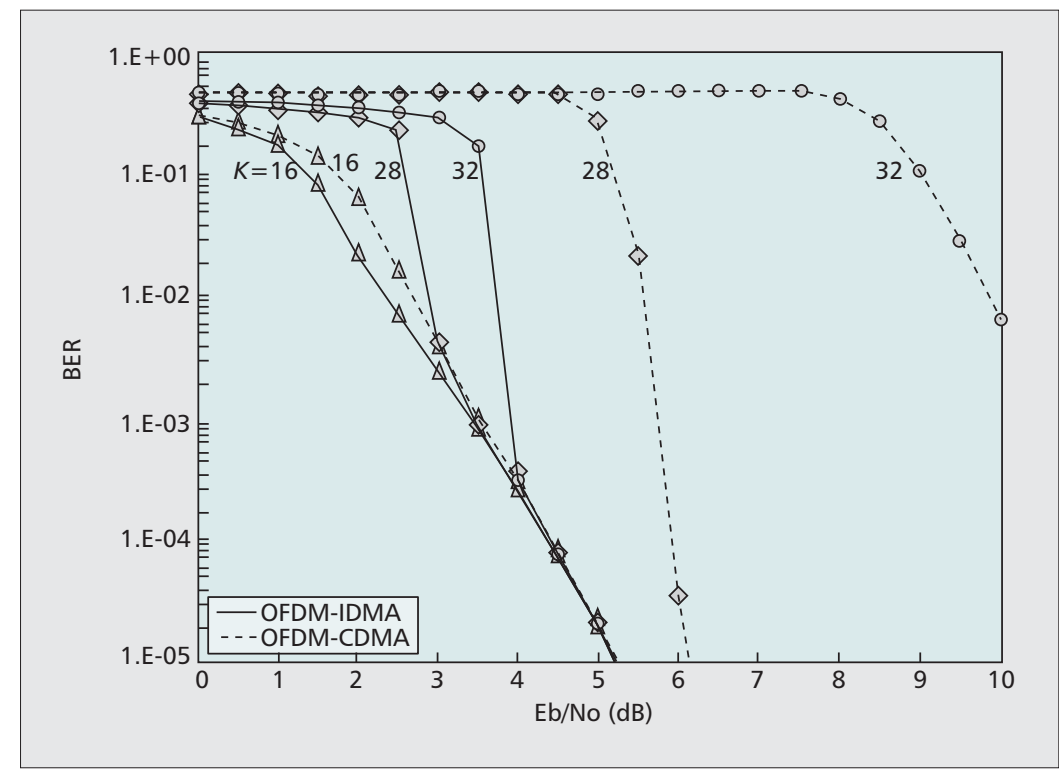

Figure 3. Performance comparison between OFDM-IDMA and OFDM$C D M A$. In both schemes, information bit length $=512$, iteration number $=5$ when $\mathrm{K}=16$, and iteration number $=15$ when $\mathrm{K}=28$ and 32 .

We will provide numerical examples to elaborate on these properties in the next section.

\section{COMPARISONS BetWEen OFDM-IDMA AND Other Alternative TeChnologies}

In what follows, we demonstrate the advantages of OFDM-IDMA over other alternatives using numerical results. We mainly consider the uplink scenario. (More research is required for downlink MUD.) Only Rayleigh fading is considered in Figs. 3 and 4. Path loss and shadow fading are considered in Fig. 5.

We assume that every user transmits at an 
equal rate $r=R / K$ bits per chip. Here a chip is a basic unit $x_{k}(n)$ in Fig. 1, and $R$ is the system aggregate rate. The number of OFDM subcarriers is 512. For simplicity, we always assume that the Rayleigh fading factors of different subcarriers are independent of each other [10]. Also for simplicity, we assume that the subcarrier allocation is random (without water-filling type optimization).

\section{OFDM-IDMA vs. OFDM-CDMA}

Figure 3 is a comparison between OFDM-IDMA and OFDM-CDMA based on the same rate-1/2

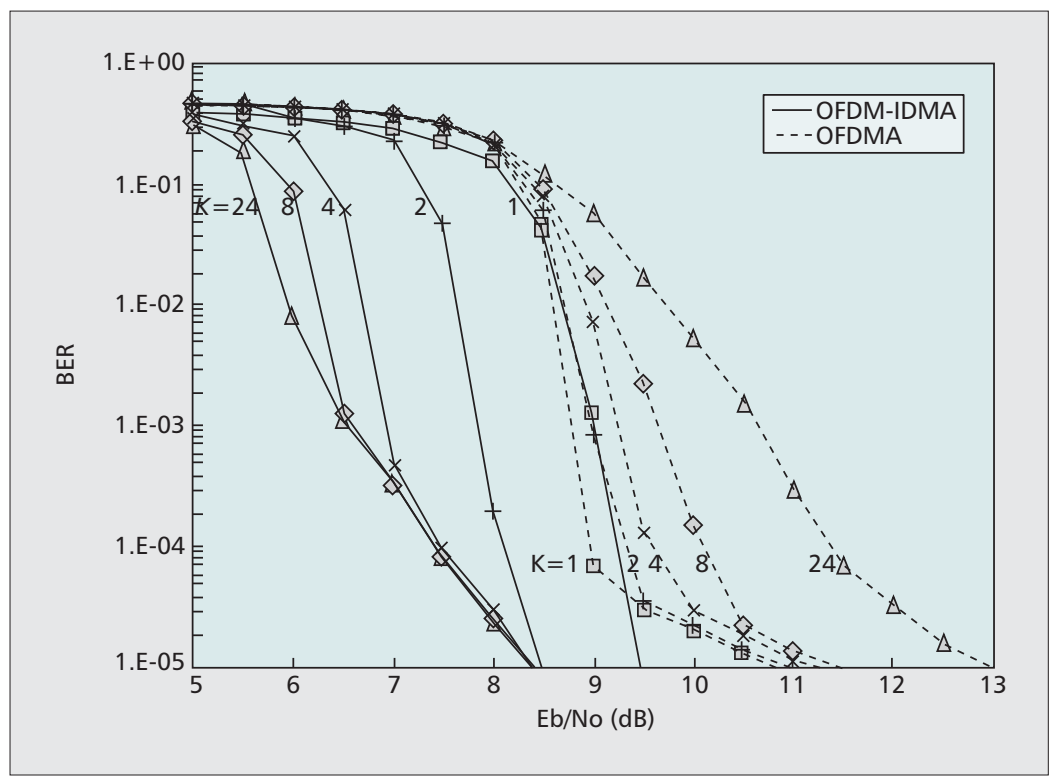

Figure 4. Performance comparison between OFDM-IDMA and OFDMA with throughput 3. The number of iterations for both schemes is 10. In OFDM-IDMA, the information block length for each code stream is 512 (i. e., 1024 coded bits after a rate-1/2 FEC coding). The total information block length for each user is $(512 \times 24) / \mathrm{K}$ for both OFDM-IDMA (with $24 / \mathrm{K}$ code streams per user) and OFDMA.

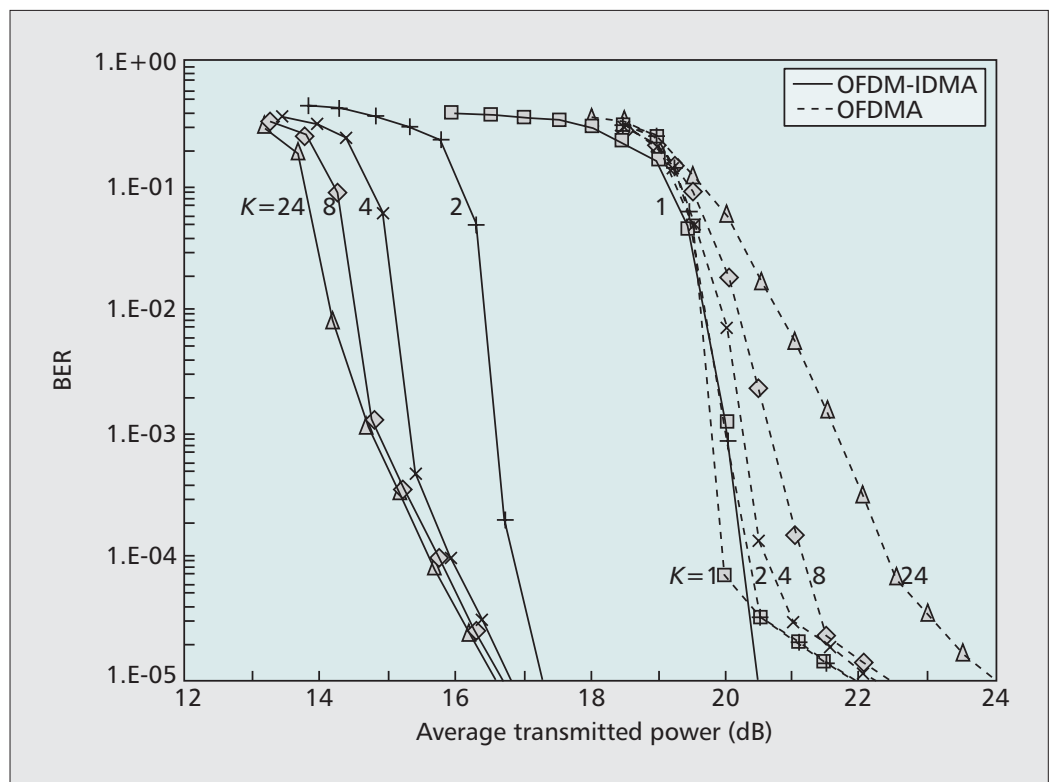

Figure 5. Performance comparison between OFDM-IDMA and OFDMA with throughput 3 in an uplink scenario with path loss and shadow fading. The system parameters are the same as those in Fig. 4. convolutional code with generator $(23,35)_{8}$ in a Rayleigh fading channel. The lengths of the repetition code in OFDM-IDMA and spreading sequence in OFDM-CDMA are both 16. QPSK modulation is used. For clarity, the path loss and shadow fading are not included in this example. In OFDM-CDMA, the spreading sequences are the orthogonal Walsh-Hadamard sequences when $K=16$ and randomly generated when $K>$ 16. The detection method (post-equalization) in [11] is employed in OFDM-CDMA. Its complexity is similar to that of OFDM-IDMA except that an extra matrix (with size $K \times K$ ) inversion is involved in its first iteration. When $K=16,28$ and 32 , the system aggregate rate $R=1,1.75$, and 2 bits/chip respectively. From Fig. 3, we can clearly see that OFDM-IDMA outperforms OFDM-CDMA and that the performance advantage increases as $K$ increases. Here, the performance gain with OFDM-IDMA is mainly attributed to the use of user-specific interleavers, which greatly facilitates the convergence of iterative MUD [2].

\section{OFDM-IDMA vs. OFDMA}

OFDM-IDMA supports flexible allocation of resources among users. Suppose that a repetition code is employed for frequency diversity. Highrate transmission can be supported by a multistream scheme with superposition coding, as illustrated in Fig. 1c where two code streams are assigned to each user. The two streams are independently interleaved using $\pi_{k}^{1}$ and $\pi_{k}^{2}$, respectively, and superimposed [8] before subcarrier allocation. Assuming the same rate for all ENCs, the information rate of a user in Fig. 1c is twice that of a user in Fig. 1b. This principle can be easily generalized to allocate more code streams to a user as required. This arrangement provides great flexibility for adjusting the rates of different users. The receiver operations are nearly the same as those in Fig. 1b, since a code stream can be simply treated as a "virtual" user at the receiver.

Figure 4 shows the performance of the worst user (with the lowest power) in an OFDMIDMA system using a rate- $1 / 2$ convolutional code (with generator $\left.(23,35)_{8}\right)$ followed by a length- 8 repetition code and QPSK modulation. The rate for each code stream is $1 / 8$. We fix the aggregate rate $R$ to 3 , so 24 code streams are involved. We assume that all users have the same number of streams. Thus each user is assigned one stream when the number of users $K=24$, and two streams when $K=12$, etc. The unequal power allocation discussed in [2] is applied. Denote by $E$ the reference power level. The power levels of the 24 streams are, respectively,

- $E$ for 13 streams

- $E+3.1677 \mathrm{~dB}$ for 2 streams

- $E+3.9596 \mathrm{~dB}$ for 2 streams

- $E+5.5430 \mathrm{~dB}$ for 7 streams

The average $E_{b} / N_{0}$ in Fig. 4 is computed based on $E$ and the above power profile.

For comparison, we show the performance of an OFDMA system (with the same throughput 3 ) based on the BICM scheme using 16-QAM modified set partitioning mapping [14]. (It can 
be shown that the detection complexity of OFDMA with BICM considered here is comparable to that of OFDM-IDMA.) We did not include the results for OFDM-CDMA due to its difficulty in achieving a high single-user rate.

We only consider Rayleigh fading in Fig. 4 (i.e., no path loss and shadow fading). It can be seen that OFDM-IDMA has similar performance to OFDMA for $K=1$. In this case, all 24 code streams are assigned to the sole user, hence all streams experience the same fading (i.e., for a fixed $n$, all $\left\{H_{k}(n), \forall k\right\}$ are the same in Eq. 2). Interestingly, this represents a relatively bad environment for the $\mathrm{CBC}$ detector, as we can observe that the OFDM-IDMA performance improves as $K$ increases. It appears that when $K$ increases, the fluctuation among $H_{k}(n)$ (caused by the independent channel fading of different users) for different $k$ is beneficial to the CBC detector. In contrast, the performance of OFDMA degrades as $K$ increases. ${ }^{4}$ Clearly, when $K$ is large, OFDM-IDMA significantly outperforms OFDMA.

OFDM-IDMA employing superposition coding also provides a convenient method for waterfilling-based resource allocation, which can be realized simply by varying the numbers of code streams on each subcarrier, and the adjustment can be made very fine by employing low-rate codes. As a comparison, water-filling using conventional coded modulation schemes can be a quite difficult issue: a large collection of different encoders/decoders may be needed if we want a large rate range and/or a fine adjustment step size for water-filling.

We now proceed to examine the near-far effect and shadow fading in a single hexagon cell environment. The channels considered in Fig. 5 include three factors: path loss, shadow fading, and Rayleigh fading. For path loss, the fourth power law is used and the distance from the farthest corner of the cell to the base station is normalized to 1 . For shadow fading, a lognormal random variable with standard deviation of $8 \mathrm{~dB}$ is used. The AWGN is complex with variance $1 / 2$ per dimension. We assume that the transmitter has knowledge of the path loss and shadow fading factors and we allow an outage probability of 0.01 . The power allocation and channel matching strategies in [2] are used. Figure 5 shows the BER performance of the worst user versus the average total transmitted power. The performance of an OFDMA scheme with BICM is also given as reference.

It can be observed from Fig. 5 that the performance of OFDMA degrades with increasing $K$ (the reason is the same as that explained in footnote 5). In contrast, the power efficiency of OFDM-IDMA improves as $K$ increases. The difference between the two systems is related to the so called "multi-user gain" and its theoretical explanation can be found in [7] and [15]. ${ }^{5}$ Figure 6 provides an illustration of multi-user gain. The required average transmitted sum power versus system throughput for different multiple access schemes is shown in this figure. For simplicity, we only consider quasi-static flat fading channels here. We can see from this figure that there are significant gaps between the theoretical limit and the required minimum transmitted sum power of

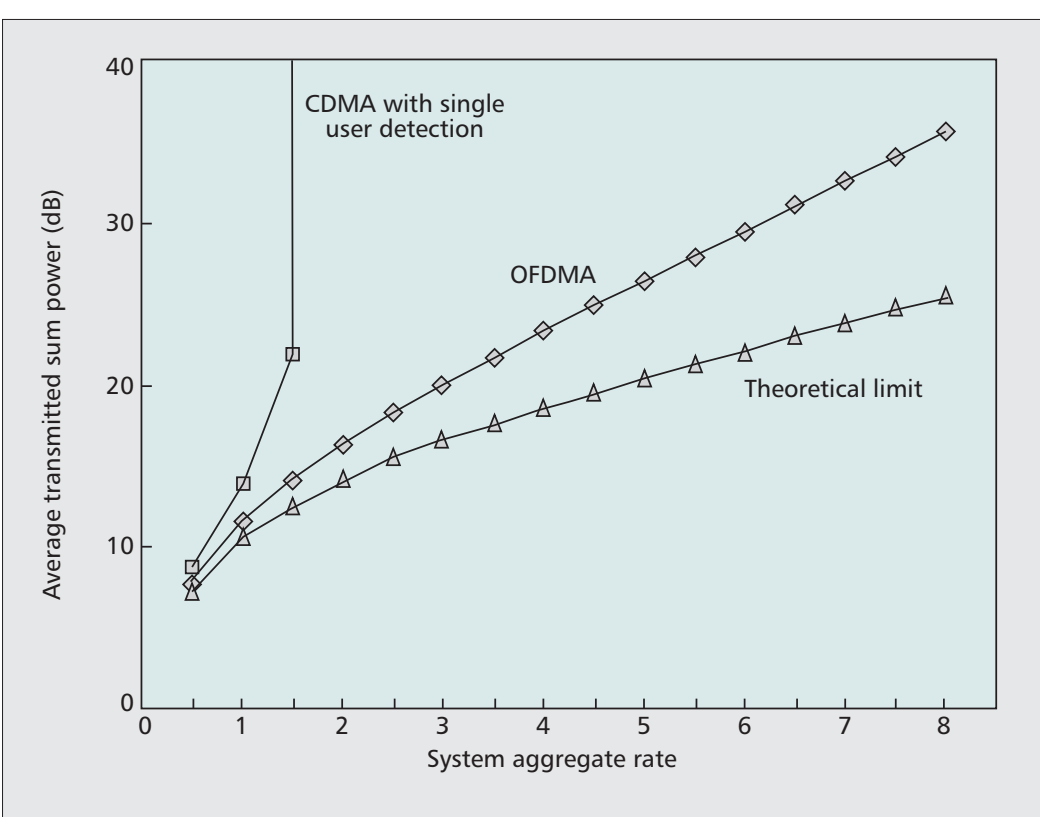

Figure 6. The average transmitted sum power versus system aggregate rate for different multiple access schemes in a single-cell environment. The channel conditions are the same as those in Fig. 5 except that flat fading channels are considered in this figure. The user number $\mathrm{K}=4$.

OFDMA and conventional (random waveform) CDMA with single user detection. The theoretical limit in Fig. 6 can only be achieved by schemes with multi-user transmission and detection [15]. Although only flat fading channels are considered in this figure, the trend is the same for frequency selective channels, which is demonstrated by the simulation results in Fig. 5. From Fig. 5 , for $K=4$, OFDM-IDMA can achieve about $4 \mathrm{~dB}$ gain compared with OFDMA, which is in line with the relative gap between the theoretical limit and the performance of OFDMA in Fig. 6. Clearly, the impact of multi-user gain is very significant, and OFDM-IDMA provides a practical means toward the realization of such gain.

\section{DISCUSSIONS AND CONCLUSIONS}

We have outlined the basic principles of OFDMIDMA and compared it with other alternatives, including OFDM-CDMA and OFDMA. We have shown that OFDM-IDMA has significant advantages regarding spectral and power efficiency, flexibility for adaptive transmission, and receiver complexity.

As a relatively new concept, there remain many outstanding issues in OFDM-IDMA, including pilot signal design, channel estimation, close-loop power control, adaptive transmission, and resource allocation. The possibility of lowcost MUD creates quite different dimensions to these issues compared to the situation in traditional systems as many functions can now be conducted jointly and iteratively.

Our discussion here relates mainly to the uplink. Theoretically, multi-user gain also applies to the downlink [15], but the related MUD cost can be a serious concern. From Fig. 5, a large portion of the potential multi-user gain can be achieved by supporting only a small number of
${ }^{4}$ The performance of OFDMA is not affected by $K$ if an ideal code is employed, but this is not the case for the practical BICM scheme used here. When Kincreases, the block length decreases and the BICM performance degrades. (As stated in the caption of Fig. 4, the total information block length for each user is $(512 \times 24) / K$ for both OFDM-IDMA and OFDMA.)

${ }^{5}$ The multi-user gain here is related to the discussion in section 6.2.2 in [15] on multiple access fading channels. It is subtly different from the multi-user diversity gain discussed in [15] for opportunistic communication. achieving the capacity of 


As a relatively new
concept, there
remain many
outstanding issues
in OFDM-IDMA
including pilot signal
design, channel
estimation,
close-loop power
control, adaptive
transmission and
resource allocation.

active users (e.g., $K=4$ ). Although this observation is made for the uplink in Fig. 5, it is also true for the downlink following the uplink/downlink duality principle [15]. The available gain can outweigh the cost concern (since MUD for a small $K$ is less demanding) and further research on this issue is desirable.

In conclusion, OFDM-IDMA appears a competitive candidate for future wireless communication systems.

\section{ACKNOWLEDGMENT}

The authors wish to thank Mr. Yueqian Li for his help in the numerical simulations.

\section{REFERENCES}

[1] C. Berrou and A. Glavieux, "Near Shannon Limit Error Correcting Coding and Decoding: Turbo-Codes," IEEE Trans. Commun., vol. 44, Oct. 1996, pp. 1261-71.

[2] L. Liu, J. Tong, and Li Ping, "Analysis and Optimization of CDMA Systems with Chip-Level Interleavers," IEEE JSAC, vol. 24, Jan. 2006, pp. 141-50.

[3] H. Schoeneich and P. A. Hoeher, "Adaptive Interleave Division Multiple Access - A Potential Air Interface for $4 \mathrm{G}$ Bearer Services and Wireless LANs," Proc. WOCN 2004, Muscat, Oman, June 2004, pp. 179-82.

[4] K. Kusume and G. Bauch, "CDMA and IDMA: Iterative Multiuser Detections for Near-Far Asynchronous Communications," IEEE Int'l. Symp. Personal, Indoor, and Mobile Radio Commun. (PIMRC'05), Berlin, Germany, vol. 1, Sept. 2005, pp. 426-31.

[5] I. Mahafeno, C. Langlais, and C. Jego, "OFDM-IDMA Versus IDMA with ISI Cancellation for Quasi-Static Rayleigh Fading Multipath Channels," Proc. 4th Int'l. Symp. Turbo Codes \& Related Topics, Munich, Germany, Apr. 3-7, 2006

[6] S. Zhou et al., "Novel Techniques to Improve Downlink Multiple Access Capacity for Beyond 3G، "IEEE Commun. Mag., vol. 43, Jan. 2005, pp. 61-69.

[7] P. Wang, J. Xiao, and Li Ping, "Comparison of Orthogonal and Non-Orthogonal Approaches to Future Wireless Cellular Systems," IEEE Vehic. Tech. Mag., vol. 1, no. 3, Sept. 2006, pp. 4-11.

[8] J. Tong, Li Ping, and X. Ma, "Superposition Coding with Peak-Power Limitation," Proc. IEEE Int'l. Conf. Commun. (ICC'06), Istanbul, Turkey, 11-15 June 2006, pp. $1718-23$.

[9] N. Yee, J.-P. Linnartz, and G. Fettweis, "Multicarrier CDMA in Indoor Wireless Radio Networks," Proc. IEEE
Int'l. Symp. Personal, Indoor, and Mobile Radio Commun. (PIMR'93), Yokohama, Japan, Sept. 1993, pp. 109-13.

[10] K. Fazel and S. Kaiser, Multi-Carrier and Spread Spectrum Systems, Chichester: John Wiley \& Sons, 2003.

[11] I. Cosovic et al., "Performance of Coded Uplink MCCDMA with Combined-Equalization in Fading Channels," Proc. IST Mobile \& Wireless Commun. Summit (IST'04), Lyon, France, June 2004, pp. 692-96.

[12] L. Hanzo et al., OFDM and MC-CDMA for Broadband Multi-user Communications, WLANs and Broadcasting, John Wiley \& Sons, 2003.

[13] X. Wang and H. V. Poor, "Iterative (Turbo) Soft Interference Cancellation and Decoding for Coded CDMA," IEEE Trans. Commun., vol. 47, July 1999, pp. 1046-61.

[14] J. Tan and G. L. Stüber, "Analysis and Design of Symbol Mappers for Iteratively Decoded BICM," IEEE Trans. Wireless Commun., vol. 4, Mar. 2005, pp. 662-72.

[15] D. Tse and P. Viswanath, Fundamentals of Wireless Communication, Cambridge: Cambridge University Press, 2005.

\section{BIOGRAPHIES}

LI PING [S'87, M'91] (eeliping@cityu.edu.hk) received his Ph.D. degree at Glasgow University in 1990. He lectured at the Department of Electronic Engineering, Melbourne University, from 1990 to 1992, and worked as a member of research staff at Telecom Australia Research Laboratories from 1993 to 1995. He has been with the Department of Electronic Engineering, City University of Hong Kong, since January 1996, where he is now a chair professor. His research interests are mixed analog/digital circuits, communications systems, and coding theory. He was awarded a British Telecom-Royal Society Fellowship in 1986, the IEE J. J. Thomson premium in 1993, and a Croucher Senior Research Fellowship in 2005.

QINGHUA GUo (qh.guo@student.cityu.edu.hk) received his B.E. degree in electronic engineering and M.E. degree in signal and information processing from Xidian University, China, in 2001 and 2004, respectively. He is currently working toward the Ph.D. degree at City University of Hong Kong. His research interests include statistical signal processing and broadband wireless communications.

JUN TONG [S'06] (jun.tong@student.cityu.edu.hk) received the B.E degree in information engineering and the M.E. degree in signal and information processing from the University of Electronic Science and Technology of China, Chengdu, in 2001 and 2004, respectively. He is currently working toward the Ph.D. degree at City University of Hong Kong. His research interests are signal processing and coding techniques in communication systems. 